\title{
Prevalence of Cytauxzoon felis infection in healthy cats from enzootic areas in Arkansas, Missouri, and Oklahoma
}

Theresa E Rizzi ${ }^{1 *}$, Mason V Reichard ${ }^{1}$, Leah A Cohn², Adam J Birkenheuer ${ }^{3}$, Jared D Taylor ${ }^{1}$ and James H Meinkoth ${ }^{1}$

\begin{abstract}
Background: Infection with Cytauxzoon felis in domestic cats can cause fever, lethargy, depression, inappetence, icterus, and often death. With a high mortality rate, cytauxzoonosis was historically considered a fatal disease. Within the last 15 years, cats with or without treatment have been recognized as chronically infected survivors of C. felis infection. Our objective was to determine the prevalence of C. felis in healthy domestic cats from Arkansas, Missouri, and Oklahoma.

Methods: Infection with C. felis was determined using DNA extracted from anticoagulated whole blood and PCR amplification using C. felis-specific primers. Chi-square, Fisher's exact tests, and odds ratios were used to compare proportions of cats infected with C. felis.

Results: Blood samples were collected from 902 healthy domestic cats between October 2008 and April 2012. DNA from Cytauxzoon felis was detected in 56 of 902 (6.2\%; 95\% confidence interval, 4.7-7.9) samples. The highest prevalence of C. felis infection (15.5\%; 10.3-21.7) was observed in cats from Arkansas, followed by cats from Missouri (12.9\%; 6.1-24.0), and cats from Oklahoma (3.4\%; 2.2-5.1). Cats sampled in Arkansas and Missouri were 5.1 and 4.2, respectively, times more likely to be chronically infected with C. felis than cats from Oklahoma.

Conclusions: Infection with C. felis is common in domestic cats through Arkansas, Missouri, and Oklahoma. The high prevalence of $C$. felis reported herein suggests that infected domestic cats are likely reservoirs of infection for naive felines. The high prevalence of $C$. felis substantiates the importance for the use of approved acaricides on cats to prevent cytauxzoonosis.
\end{abstract}

Keywords: Arkansas, Cats, Cytauxzoon felis, Missouri, Oklahoma

\section{Background}

Cytauxzoon felis is a tick-transmitted protozoan parasite that can cause fatal disease in domestic cats and some wild captive felids [1-5]. Cytauxzoonosis was first described in 1976 [6]. Historically, bobcats (Lynx rufus) have been considered reservoirs for C. felis and domestic cats (Felis catus) as aberrant dead-end hosts [7-10]. More recently, a study demonstrated transmission of $C$. felis from chronically infected domestic cats to naive cats via tick bite, demonstrating cats are competent reservoirs for $C$. felis [11,12].

\footnotetext{
* Correspondence: theresa.rizzi@okstate.edu

'Department of Veterinary Pathobiology, Oklahoma State University,

Stillwater, OK, USA

Full list of author information is available at the end of the article
}

Experimental transmission of $C$. felis has been demonstrated with Dermacentor variabilis $[8,11]$ and Amblyomma americanum [11,12]. The occurrence of cytauxzoonosis coincides with the distribution and seasonal activity of $A$. americanum [11], possibly explaining why cytauxzoonosis is not present in domestic cats in regions where C. felis is present in bobcats but A. americanum are not found $[9,15]$. Cytauxzoonosis is enzootic in the south-central United States, but cases have been identified in states extending to the mid-Atlantic coast [16-18].

Onset of disease typically follows $10-14$ days after $C$. felis-infected ticks feed on naive cats. Cats become depressed, anorectic, markedly pyretic, dehydrated, pale, and icteric as schizont-laden macrophages occlude vasculature. Most cats die within a week of initial illness. 
Treatments for cytauxzoonosis have been tried with limited success $[19,21,22]$. A recent study demonstrated a $60 \%$ survival in cats given the combination of atovaquone and azithromycin plus supportive care [22], historically the disease was believed to be nearly always fatal with few reported survivors $[19,20,23]$.

In addition to cats that survive illness and can remain chronically infected, chronically infected cats have been recognized with no known antecedent illness [23-25]. Chronically infected cats have been reported in northwestern Arkansas and north-eastern Oklahoma [23,24], Florida and Tennessee [25], and Arkansas and Georgia [26]. We hypothesized that chronically infected cats might serve as an additional reservoir of $C$. felis for naive domestic cats. Because domestic cats are more likely to live near other domestic cats than near bobcats, these reservoir cats might assume an important role in disease transmission. The purpose of the current study was to determine the prevalence of $C$. felis infection in domestic cats in an enzootic area with high incidence of disease.

\section{Methods}

Participation in this survey was solicited from veterinarians in Oklahoma, Missouri and Arkansas (Figure 1). Whole blood samples collected in EDTA from domestic cats for routine procedures or illness unrelated to cytauxzoonosis at private veterinary clinics, animal shelter/spay/ neuter programs, or client cared for feral cats in Oklahoma, Missouri and Arkansas were submitted for this study from October 2008 through April 2012. The samples were used for other blood testing prior to submission for this study. Criteria for inclusion were domestic cats at least 6 months of age that were not exhibiting signs of illness consistent with $C$. felis infection. Cats previously diagnosed with $C$. felis infections were excluded from the study. All blood samples submitted were stored at $4^{\circ} \mathrm{C}$ up to 6 months until shipment to North Carolina State University for testing.

Blood samples were analyzed for $C$. felis infection using previously described methods [25]. Briefly, DNA was extracted from whole blood using the QIAmp DNA Blood Mini Kit or Magattract DNA Blood Mini M48 Kit (Qiagen Inc., Valencia, CA). Amplification of a portion of the $18 \mathrm{~S}$ rRNA gene of $C$. felis was accomplished using PCR and primers specific to $C$. felis. Primer sequences were: 5'GCGAATCGCATTGCTTTATGCT-3'and 5'-CCAAATG ATACTCCGGAAAGAG-3'. Positive controls consisted of DNA extracted from known C. felis-infected cat whole blood and negative controls consisted of purified (no DNA) water. Samples that were negative for C. felis infection were screened for the presence of PCR inhibitors via amplification of a glyceraldehyde 3-phosphate dehydrogenase (GAPDH) pseudogene as previously described [27]. Sample processing, DNA extraction, master mix assembly, PCR amplification, and post amplification processing were performed in separate areas to avoid amplicon contamination. Good laboratory procedures were employed to ensure uniformity, consistency, reliability, and reproducibility of results.

The prevalence of $C$. felis infection in cats was calculated according to Bush et al. [28]; 95\% confidence intervals were

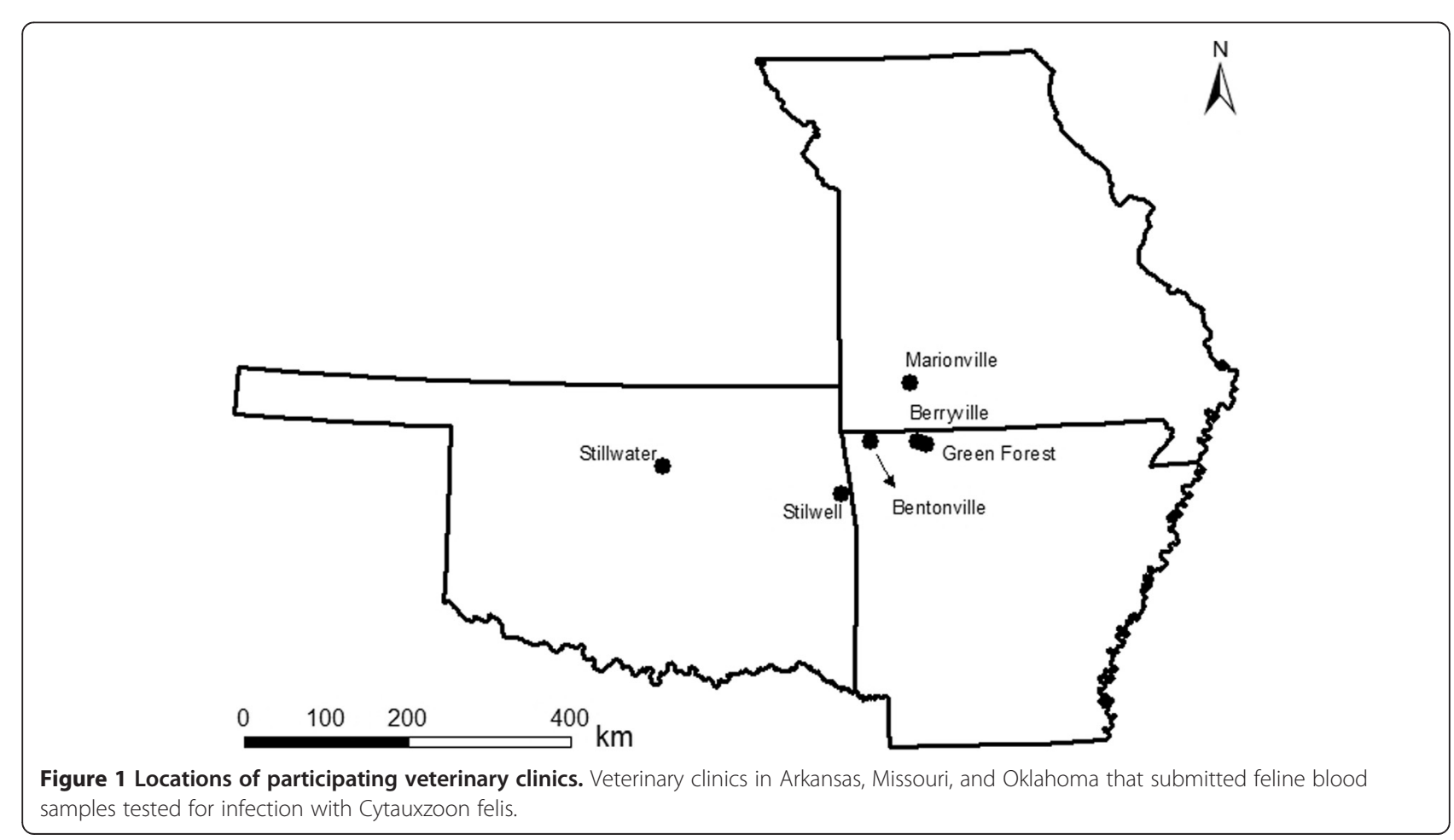


calculated according to Sterne's exact method [29] using Quantitative Parasitology 3.0 [30]. Proportions of cats infected with $C$. felis were compared with Chi-square and Fisher's exact tests using Sigma Plot 12.5 (Systat Software Inc., San Jose, CA). Odd ratios [31] were calculated to express differences in the proportion of cats infected from Arkansas, Missouri, and Oklahoma.

\section{Results}

A total of 902 feline blood samples were tested from 2008-2012:161 from AR, 62 from MO, and 679 from OK (Table 1). Of these 902 samples, C. felis was detected in 56 cats, resulting in an overall prevalence $(95 \%$ confidence interval) of $6.2 \%(4.7-7.9)$. Of the 161 cats collected in AR, C. felis was detected in 25, resulting in a prevalence of $15.3 \%$ (10.3-21.7). Of the 62 cats collected in $\mathrm{MO}, \mathrm{C}$. felis was detected in 8 , resulting in a prevalence of $12.9 \%(6.1-24.0)$. Of the 679 cats collected in $\mathrm{OK}, \mathrm{C}$. felis was detected in 23 , resulting in a prevalence of $3.4 \%(2.2-5.1)$. Statistically discernable differences $\left(X^{2}=37.458, \mathrm{df}=2, \mathrm{P}=<0.0001\right)$ were detected in the proportion of cats infected with C. felis in AR, MO, and OK. The prevalence of $C$. felis infected cats in AR (15.3\%) and MO (12.9\%) were statistically higher $\left(X^{2}=32.730\right.$, $\mathrm{df}=1, \mathrm{P}=<0.0001 ; X^{2}=10.570, \mathrm{df}=1, \mathrm{P}=0.001$, respectively) than that of cats collected from OK (3.4\%). Cats sampled in AR and MO were 5.1 and 4.2, respectively, times more likely to be infected with $C$. felis than cats from OK.

Four community veterinary practices (clinics A, B, C, and $\mathrm{D}$ ) in AR contributed samples with most obtained from client-owned cats (Table 1). All four of these clinics are located within approximately a 60 miles radius of each other in the north-western part of AR. All four clinics reported treating many cats with both fatal and non-fatal cytauxzoonosis throughout the years. Blood samples of 71 cats were submitted from clinics $A$ and B in Bentonville, AR with 2 cats infected with C. felis. Thirty samples were submitted from clinic $C$ in Berryville, AR with 4 cats testing positive (13.3\%).
Clinic D, located in Green Forest, AR (approximately 10 miles east of Berryville) submitted 60 samples with 18 testing positive (30.0\%). The prevalence of C. felis in cats from Arkansas clinics were statistically discernible $\left(X^{2}=18.030, \mathrm{df}=3, \mathrm{P}=0.0004\right)$ from each other. Multiple comparisons of the proportion of cats infected with $C$. felis demonstrated the prevalence of $C$. felis in cats from Clinic D (30\%) was statistically higher $\left(X^{2}=14.331, \mathrm{df}=2\right.$, $\mathrm{P}=<0.001)$ than those from Clinic B (3.2\%).

Sixty-two cat samples from Marionville, MO all came from a single, client-managed outdoor population (Table 1). The client reported deaths attributed to cytauxzoonosis within this population of cats in the past. Eight samples tested positive by PCR (12.9\%).

A total of 679 blood samples were submitted from veterinary practices and animal shelters located in central and eastern OK (Table 1). Of the 679 samples, 23 were positive by PCR for $C$. felis (3.4\%). The prevalence of $C$. felis in cats from Oklahoma clinics were statistically discernible $\left(X^{2}=48.737, \mathrm{df}=2, \mathrm{P}=<0.0001\right)$ from each other. Multiple comparisons of the proportion of cats infected with $C$. felis demonstrated the prevalence of $C$. felis in cats from Clinic G (16.9\%) was statistically higher $\left(X^{2}=27.969, \mathrm{df}=1, \mathrm{P}=<0.001 ; X^{2}=28.348, \mathrm{df}=1\right.$, $\mathrm{P}=<0.001$ than those of Clinics $\mathrm{F}(2.0 \%)$ and $\mathrm{H}(1.2 \%)$, respectively. Thirteen of the 77(16.9\%) blood samples from Stillwell, OK (eastern OK approximately seven miles from the Arkansas border) were infected with C. felis. The majority of samples collected in OK were from the Stillwater area (central OK). Prior to the current study, relatively few cats subclinically infected with $C$. felis were known from central OK.

\section{Discussion}

This is the first study to document the prevalence of chronic C. felis infection in domestic cats in a region of the United States with a high incidence of clinical cytauxzoonosis. The prevalence of C. felis $(6.2 \%)$ in the current study is higher than the previously reported $0.3 \%$ in free roaming cats in Florida, North Carolina and

Table 1 Prevalence of Cytauxzoon felis in cats collected in Arkansas, Missouri, and Oklahoma from 2008-2012

\begin{tabular}{lllll}
\hline State & Sample origin & Number of samples & Number of cats infected & Prevalence (95\% Cl) \\
\hline Arkansas & Clinic A (Bentonville) & 8 & 1 & $12.5 \%(0.6-50.0)$ \\
& Clinic B (Bentonville) & 63 & 2 & $3.2 \%(0.5-10.8)$ \\
& Clinic C (Berryville) & 30 & 4 & $13.3 \%(4.7-29.8)$ \\
& Clinic D (Green Forest) & 60 & 18 & $30.0 \%(19.6-42.9)$ \\
Missouri & Clinic E (Marionville, MO) & 62 & 8 & $12.9 \%(6.1-24.0)$ \\
Oklahoma & Clinic F (Stillwater \& surrounding areas) & 349 & 7 & $2.0 \%(1.0-4.1)$ \\
& Clinic G (Stillwell) & 77 & 13 & $16.9 \%(9.7-27.2)$ \\
& Clinic H (Stillwater) & 253 & 3 & $1.2 \%(0.3-3.5)$ \\
Total & & 902 & 56 & $6.2 \%(4.7-7.9)$ \\
\hline
\end{tabular}


Tennessee [25]. In addition, there were overall differences in the prevalence of C. felis in cats among Arkansas, Missouri, and Oklahoma with statistically discernible differences in the prevalence of $C$. felis in cats from AR and $\mathrm{OK}$.

Cytauxzoonosis is common in this area of the country from spring to early fall $[32,33]$. There have been reports of domestic cats either recovering from clinical infection or being parasitemic without evidence of clinical disease [8,16,20,21,23-25,35,36,38]. Meinkoth et al. [24] identified 18 domestic cats that survived natural infection with C. felis in eastern OK (Catoosa and Tahlequah) and north-west AR (Berryville, Fayetteville, Clarksville). They concluded treatment was not a likely explanation for the cats' survival because some cats had no treatment, and others were treated with therapies not effective against C. felis. Possible explanations for survival include strain variation in virulence, innate immunologic response, or variations in tick inoculum. Strain variation is unlikely to be the entire explanation as many of the healthy, chronically infected cats were from households and neighborhoods where lethal cytauxzoonosis was also reported.

The prevalence of C. felis in apparently healthy cats from limited geographic areas suggests that there may be less virulent strains of C. felis. In 2009, C. felis isolates from blood samples obtained from 88 domestic cats in Georgia and Arkansas were analyzed. An association between infections with $C$. felis containing particular internal spacer regions (ITS) and survival rates of infected cats was noted in addition to genotype variability from samples originating in AR and GA [34]. However, in a subsequent study the ITS regions in asymptomatic cats were found to be the same as ITS regions previously detected in mostly fatal infections. The investigators concluded that analyzing ITS regions, while suitable for studying geographic variability, was not useful in determining pathogenicity of the strains [26]. Additional research will be required to identify genetic markers reflective of the virulence of $C$. felis strains.

It has been postulated cats surviving cytauxzoonosis could serve as reservoirs of infection for competent vectors $[11,24,35]$, as cats remain parasitemic for extended periods $[12,26,36]$. The relative significance of such a reservoir would depend on the number of chronically infected cats, which historically was assumed to be low. The results of this study would support the contention that these cats could represent a substantial reservoir. The widespread occurrence of cats subclinically infected with $C$. felis in disparate areas suggests that chronically infected cats are present and available as reservoirs of infection across geographic regions.

Reichard et al. demonstrated that virulent infection could be transmitted from a chronically infected domestic cat to another cat via $A$. americanum vector [11]. A survivor of natural infection with $C$. felis was used to acquisition feed $A$. americanum nymphs. The $C$. felisinfected nymphs were allowed to molt to adults, which successfully transmitted $C$. felis to a naive cat. The recipient cat experienced clinical disease, but survived infection. In a subsequent transmission trial [12], similar cat-to-cat transmission was confirmed when A. americanum fed on the donor cat from the previous transmission trial were able to induce clinical cytauxzoonosis in four cats [13]. These transmission trials not only confirmed $A$. americanum as a tick vector for $C$. felis, but that cats with subclinical disease could be reservoirs of infection for naive domestic cats.

There have been reports of cytauxzoonosis survivor cats resistant to challenge with virulent C. felis $[19,20]$, unpublished observation]. It is possible that immune response to initial infection of C. felis in chronically infected cats is sufficient to protect against subsequent challenge. Further research is necessary to determine the possibility of developing biologics that offer passive immunity to cats with clinical cytauxzoonosis or protection via immunization [36].

Cats with subclinical cytauxzoonosis may be persistently parasitemic for prolonged periods [37]. Since inclusion in this study, additional follow-up has become available for some cats, with several remaining parasitemic for years. In one cat undergoing treatment for intestinal lymphoma, the number of erythrocytes containing piroplasms as seen on blood film analysis increased after initiation of chemotherapy, suggesting immunosuppression may exacerbate parasitemia. To the authors' knowledge, no cats recrudesced with cytauxzoonosis since recognition of subclinical infection.

With the realization that domestic cats are competent reservoirs of $C$. felis infection, information regarding the prevalence of carriers becomes increasingly important. Cats at highest risk of being infected with $C$. felis include those whose home ranges are in low density residential areas, along urban edges, with access to wooded cover, and in close proximity to natural or unmanaged habitat, all of which may lead to closer proximity to ticks that have fed on bobcats [32]. However, domestic cat reservoirs for the pathogen may not only be more likely to live in close proximity to other domestic cats than would bobcats, but they may also be moved during owner relocation to geographic areas where C.felis has not been endemic. Any region with $A$. americanum or $D$. variabilis could potentially allow for transmission of pathogen from these carrier cats. Because the geographic range of A. americanum has dramatically expanded in recent years [38], this could potentially lead to infection in areas where it has not been previously seen.

The high prevalence of C. felis in enzootic areas emphasizes the need to use measures to prevent tick exposure 
both to carrier cats that might act as reservoirs, and to naive cats that might be exposed to infection. Unfortunately, acaricides for use on cats are limited. Flumethrin, a synthetic pyrethroid safe to use on cats, has demonstrated efficacy for preventing the transmission of $C$. felis by $A$. americanum [39] and is labelled to repel and kill D. variabilis. Keeping cats indoors, combined with regular use of flumethrin, offers the best protection both against infection of naive cats, and transmission of C. felis from chronically infected carriers.

\section{Conclusions}

In recent years domestic cats with or without treatment have been recognized as chronically infected survivors of C. felis infection. Our study demonstrates a prevalence of C. felis infection in domestic cats of $6.2 \%$ in an area of the United States with a high incidence of clinical cytauxzoonosis. Since recent studies have demonstrated domestic cats are competent reservoirs for $C$. felis and are more likely to live in close proximity to other domestic cats than would bobcats, the prevalence of infected cats will likely increase. Additionally, because of owner relocation, C. felis may be introduced in geographic areas with A. americanum or D. variabilis where C.felis is not endemic. Cytauxzoonosis is often diagnosed by observing piroplasms within erythrocytes during blood film evaluation. Since cats with subclinical disease can remain parasitemic for prolonged periods, chronic carriers presenting for any febrile illness may be mistakenly diagnosed with acute cytauxzoonosis. Because PCR would also be positive in these cats, it cannot be used to distinguish between carrier and acute infection. Prevention remains paramount by keeping cats indoors combined with regular use of flumethrin.

\section{Competing interests}

The authors declare that they have no competing interests.

\section{Authors' contributions}

TER and JHM conceived the study and participated in its design and coordination. AJB carried out molecular studies. MVR and JDT performed statistical analysis. All authors read and approved the final version of the manuscript. TER drafted the first version of the manuscript and all authors read, contributed to its content, and approved the final version of the manuscript.

\section{Acknowledgements}

Blood samples were submitted and organized through Oklahoma State University. Molecular testing occurred at North Carolina State University. Funding was provided through an internal grant by The Department of Veterinary Pathobiology, Center for Veterinary Health Science, Oklahoma State University.

The authors are grateful to cat owners and Dr. Blaine Andrew from Town \& Country Veterinary Clinic, Green Forest, AR; Drs. Tina Cole, Alan Hunnicutt and Kameron Worley from Berryville Veterinary Clinic, Berryville, AR; Dr. Paula Brown from Rose Animal Clinic, Bentonville, AR; Dr. Andrea Strecker from Oakwood Pet Hospital, Bentonville, AR; Dr. Yolanda Burton from Patterson Animal Hospital, Stillwell, OK; Dr. Barbara Dunn from Family Pet Clinic, Ardmore, OK; Drs. Annette Cowell and Sarah Peakheart from The Cat Clinic of Stillwater, Stillwater, OK, Drs. Lesa Stabbaus and Katrina Meinkoth from the Center for Veterinary Health Science at Oklahoma State University, Shelter
Medicine, Stillwater, OK who submitted samples used in this study. Ms. Victoria Catto and Mr. Henry Marr, North Carolina State University, for technical support; Dr. Kristen Baum, Department of Zoology at Oklahoma State University for providing the map used in Figure 1.

\section{Author details}

${ }^{1}$ Department of Veterinary Pathobiology, Oklahoma State University, Stillwater, OK, USA. ${ }^{2}$ Department of Veterinary Medicine and Surgery, University of Missouri, Columbia, MO, USA. ${ }^{3}$ College of Veterinary Medicine, North Carolina State University, Raleigh, NC, USA.

Received: 12 September 2014 Accepted: 20 December 2014 Published online: 08 January 2015

\section{References}

1. Garner MM, Lung NP, Citino S, Greiner EC, Harvey JW, Homer BL: Fatal cytauxzoonosis in a captive-reared white tiger (Pathera tigris). Vet Pathol 1996, 33:82-86.

2. Jakob W, Wesemeier $\mathrm{HH}$ : A fatal infection in a bengal tiger resembling Cytauxzoonosis in domestic Cats. J Comp Path 1996, 114:439-444.

3. Rotstein DS, Taylor SK, Harvey JW, Bean J: Hematologic effects of Cytauxzoonosis in Florida panthers and texas cougars in Florida. J Wildl Dis 1999, 35:613-617.

4. Harvey JW, Dunbar MR, Norton TM, Yabsley MJ: Laboratory findings in acute Cytauxzoon felis infection in Cougars (Puma concolor couguar) in Florida. J Zoo Wildl Med 2007, 38:285-291.

5. Lewis KM, Cohn LA, Downey ME, Whitney MS, Birkenheuer AJ: Evaluation of Cytauxzoon felis infection status in captive-born wild felids housed in an area endemic for the Pathogen. J Am Vet Med Assoc 2012, 241:1088-1092.

6. Wagner JE: A fatal Cytauxzoonosis-like disease in Cats. J Am VetMed Assoc 1976, 168:585-588.

7. Blouin EF, Kocan AA, Glenn BL, Kocan KM: Transmission of Cytauxzoon felis kier, 1979 from Bobcats, Felis rufus (Schreber), to domestic Cats by Dermacentor variablis (Say). J Wildl Dis 1984, 20:241-242.

8. Kier AB, Wagner JE, Morehouse LG: Experimental transmission of Cytauxzoon felis from Bobcats (Lynx rufus) to domestic Cats (Felis domesticus). Am J Vet Res 1982, 43:97-101.

9. Birkenheuer AJ, Marr HS, Warren C, Acton AE, Mucker EM, Humphreys JG, Tucker MD: Cytauxzoon felis infections are present in Bobcats (Lynx rufus) in a region where Cytauxzoonosis is not recognized in domestic Cats. Vet Parasitol 2008, 153:126-130.

10. Glenn BL, Kocan AA, Blouin EF: Cytauxzoonosis in Bobcats. J Am Vet Med Assoc 1983, 183:1155-1158.

11. Reichard MV, Meinkoth JH, Edwards AC, Snider TA, Kocan KA, Blouin EF, Little SE: Transmission of Cytauxzoon felis to a domestic Cat by Amblyomma americanum. Vet Parasitol 2009, 161:110-115.

12. Reichard MV, Edwards AC, Meinkoth JH, Snider TA, Meinkoth KR, Heinz RE, Little SE: Confirmation of Amblyomma americanum(Acari: Ixodidae) as a vector for Cytauxzoon felis (Piroplasmorida: Theileriidae) to domestic Cats. J Med Entomol 2010, 47:890-896.

13. Glenn BL, Rolley RE, Kocan AA: Cytauxzoon-like piroplasms in erthrocytes of wild-trapped bobcats in Oklahoma. J Am Vet Med Assoc 1982, 181:1251-1253.

14. Kocan AA, Kocan KM, Blouin EF, Mukolwe SW: A redescription of schizogony of Cytauxzoon felis in the Domestic Cat. Ann N Y Acad Sci 1992, 653:161-167.

15. Shock BC, Murphy SM, Patton LL, Shock PM, Olfenbuttel C, Beringer J, Prange S, Grove DM, Peek M, Butfiloski JW, Husghes DW, Lockhart JM, Bevins SN, VandeWoude S, Crooks KR, Nettles VF, Brown HM, Peterson DS, Yabsley MJ: Distribution and prevalence of Cytauxzoon felis in Bobcats (Lynx rufus), the natural reservoir, and other wild felids in Thirteen States. Vet Parasitol 2011, 175:325-330.

16. Birkenheuer AJ, Le JA, Valenzisi AM, Tucker MD, Levy MG, Breitschwerdt EB: Cytauxzoon felis infection in Cats in the Mid-Altlantic States: 34 cases (1998-2004). J Am Vet Med Assoc 2006, 228:568-571.

17. Hauck WN, Snider TA, Lawrence JE: Cytauxzoonosis in a Native Louisiana Cat. J Am Vet Med Assoc 1982, 180:1472-1474.

18. Jackson CB, Fisher T: Fatal Cytauxzoonosis in a Kentucky Cat (Felis domesticus). Vet Parasitol 2006, 139:192-195.

19. Motzel SL, Wagner JE: Treatment of experimentally induced Cytauxzoonosis in Cats with Parvaquone and Buparvaquone. Vet Parasitol 1990, 35:131-138. 
20. Ferris DH: A progress report on the status of a new disease of American Cats: Cytauxzoonosis. Comp Immunol Microbiol Infects Dis 1979, 1:269-276.

21. Green CE, Latimer K, Hopper E, Shoeffler G, Lower K, Cullens F: Administration of diminazene aceturate or imidocarb dipropionate for treatement of Cytauxzoonosis in Cats. J Am Vet Med Assoc 1999, 215:497-500.

22. Cohn LA, Birkenheuer AJ, Brunker JD, Ratcliff ER, Craig AW: Efficacy of atovaquone and azithromycin or imidocarb dipropionate in Cats with Acute Cytauxzoonosis. J Vet Intern Med 2011, 25:55-60.

23. Walker DB, Cowell RL: Survival of a domestic Cat with naturally acquired Cytauxzoonosis. J Am Vet Med Assoc 1995, 206:1363-1365.

24. Meinkoth JH, Kocan AA, Whitworth L, Murphy G, Fox JC, Woods JP: Cats surviving natural infection with Cytauxzoon felis: 18 cases (1997-1998). J Vet Intern Med 2000, 14:521-525.

25. Haber MD, Tucker MD, Marr HS, Levy JK, Burgess J, Lappin MR, Birkenheuer AJ: The detection of Cytauxzoon felis in apparently healthy free-roaming Cats in the USA. Vet Parasitol 2007, 146:316-320.

26. Brown HM, Lockhart JM, Latimer KS, Peterson DS: Identification and genetic characterization of Cytauxzoon felis in asymptomatic domestic Cats and Bobcats. Vet Parasitol 2010, 172:311-316.

27. Birkenheuer AJ, Levy MG, Breitschwerdt EB: Development and evaluation of a seminested PCR for detection and differentiation of Babesia gibsoni (Asian genotype) and B. canis DNA in canine blood samples. J Clin Microbiol 2003, 41:4172-4177.

28. Bush AO, Lafferty KD, Lotz JM, Shostak AW: Parasitology meets ecology on its own terms: Margolis et al. revisited. J Parasitol 1997, 83:575-583.

29. Reiczigel J: Confidence intervals for the binomial parameter: some new considerations. Stat Med 2003, 22:611-621.

30. Rozsa L, Reiczigel J, Majoros G: Quantifying parasites in samples of hosts. J Parasitol 2000, 86:228-232.

31. Sokal RR, Rohlf FJ: Biometry. 3rd edition. New York: W. H. Freeman and Company; 1997:887.

32. Reichard MV, Baum KA, Cadenhead SC, Snider TA: Temporal occurrence and environmental risk factors associated with Cytauxzoonosis in domestic Cats. Vet Parasitol 2008, 152:314-320.

33. Mueller EK, Baum KA, Papes M, Cohn LA, Cowell AK, Reichard MV: Potential ecological distribution of Cytauxzoon felis in domestic Cats in Oklahoma, Missouri, and Arkansas. Vet Parasitol 2013, 192:104-110.

34. Brown HM, Berghaus RD, Latimer KS, Britt JO, Rakich MR, Peterson DS: Genetic variability of Cytauxzoon felis from 88 infected domestic Cats in Arkansas and Georgia. J Vet Diagn Invest 2009, 21:59-63.

35. Carli E, Trotta M, Chinelli R, Drigo M, Sinigoi L, Tosolini P, Furlanello T, Millotti A, Caldin M, Solano-Gallego L: Cytauxzoon sp. infection in the first endemic focus described in domestic Cats in Europe. Vet Parasitol 2011, 183:343-352.

36. Tarigo JL, Scholl EH, Bird DM, Brown CC, Cohn LA, Dean GA, Levy MG, Doolan DL, Trieu A, Nordone SK, Felgner PL, Vigil A, Birkenheuer AJ: A novel candidate vaccine for Cytauxzoonosis inferred from comparative Apicomplexan genomics. PLoS One 2013, 8(10):e71233.

37. Brown HM, Latimer KS, Erikson LE, Cashwell ME, Britt JO, Peterson DS: Detection of persistent Cytauxzoon felis infection by polymerase chain reaction in three asymptomatic domestic cats. J Vet Diagn Invest 2008, 20:485-488

38. Childs JE, Paddock CD: The ascendancy of Amblyomma americanum as a vector of pathogens affecting humans in the United States. Annu Rev Entomol 2003, 48:307-337.

39. Reichard MV, Thomas JE, Arther RG, Hostetler JA, Raetzel KL, Meinkoth JH, Little SE: Efficacy of an imidacloprid 10\%/Flumethrin 4.5\% collar (Seresto (R), Bayer) for preventing the transmission of Cytauxzoon felis to domestic Cats by Amblyomma americanum. J Parasitol Res 2013, 112:11-20.

\section{Submit your next manuscript to BioMed Central and take full advantage of:}

- Convenient online submission

- Thorough peer review

- No space constraints or color figure charges

- Immediate publication on acceptance

- Inclusion in PubMed, CAS, Scopus and Google Scholar

- Research which is freely available for redistribution

Submit your manuscript at www.biomedcentral.com/submit 